\title{
Análisis de las Propiedades Psicométricas de la Escala de Autoeficacia en el Trabajo en una Muestra de Personas Empleadas en Puerto Rico
}

\author{
Analysis of psychometric properties of the Self-efficacy at Work Scale in a sample \\ of people employed in Puerto Rico
}

\author{
Yahaira Román-Cabán ${ }^{1 *}$, Miguel Martínez-Lugo ${ }^{2}$, \& Ramón Rodríguez-Montalbán ${ }^{3}$ \\ 1 Universidad Carlos Albizu, San Juan, Puerto Rico. (D) https://orcid.org/0000-0003-0253-1473 \\ 2 Universidad Carlos Albizu, San Juan, Puerto Rico. (iD https://orcid.org/0000-0001-5586-2327 \\ 3 Universidad Carlos Albizu, San Juan, Puerto Rico. (D) https://orcid.org/0000-0003-2414-1943 \\ * Correspondencia: yaharoman1@gmail.com
}

Recibido: 12 enero 2021 | Aceptado: 30 marzo 2021 | Publicado: 5 abril 2021

WWW.REVISTACARIBENADEPSICOLOGIA.COM

\section{Citar como:}

Román-Cabán, Y., Martínez-Lugo, M., \& Rodríguez-Montalbán, R. (2021). Análisis de las propiedades psicométricas de la Escala de Autoeficacia en el Trabajo en una muestra de personas empleadas en Puerto Rico. Revista Caribeña de Psicología, 5, e5367. https://doi.org/10.37226/rcp.v5i1.5367

\section{RESUMEN}

El objetivo de este estudio es determinar las propiedades psicométricas de la Escala de Autoeficacia en el Trabajo de Pepe et al. (2010): validez de constructo, análisis de ítems, análisis de fiabilidad y validez discriminante. El estudio consistió en un análisis secundario de datos con una muestra total de 678 personas trabajando en diversos sectores laborales en Puerto Rico. Los resultados de esta investigación demuestran, que la escala no cumple con los criterios de validez discriminante entre los factores de voluntad y compromiso. Concluimos, que uno de los factores que influye en que la escala no posea las características psicométricas adecuadas es la carencia de diferentes grados de desafío o impedimento para un rendimiento exitoso en la redacción de los ítems de la escala lo cual es recomendado por Bandura.

Palabras Claves: autoeficacia; autoeficacia en el trabajo; análisis psicométrico

\begin{abstract}
The purpose of this study is to determine the psychometric properties of the Work Self-Efficacy Scale (WSES): construct validity, item analysis, reliability analysis, and discriminant validity. This study consisted of secondary data analysis with a total sample of 678 participants working in various organizations in Puerto Rico. This research shows that the scale does not meet the criteria of discriminant validity between the factors of commitment and relational willingness. We conclude that one of the factors that influence the fact that the scale does not have good psychometric characteristics is the lack of different degrees of challenge or impediment for successful performance in writing the scale items, which Bandura recommends.
\end{abstract}

Keywords: self-efficacy; work self-efficacy; psychometric analysis 


\section{INTRODUCCIÓN}

Las organizaciones diariamente se enfrentan a múltiples desafíos que en ocasiones deben ser trabajados de manera inmediata. Estos desafíos hacen necesario que las empresas tengan una fuerza laboral con autoeficacia para poder enfrentar los cambios y las demandas laborales. Lo antes expuesto, pone de manifiesto que las personas que trabajan tendrán que ser capaces de atender múltiples y variadas tareas, adaptando sus conocimientos, habilidades y destrezas a las demandas laborales (Martínez, 2004). Por consiguiente, la autoeficacia en el trabajo se convierte en un constructo de interés para las organizaciones, ya que las creencias de las propias capacidades para organizar y ejecutar cursos de acción requeridos producirán determinados resultados (Bandura, 1997).

En el año 2010, Pepe et al. realizaron una investigación para analizar la estructura factorial e invarianza de la Escala de Autoeficacia en el Trabajo (WSES, por sus siglas en inglés) en algunos contextos laborales de España e Italia. Aunque la escala se encuentra redactada en el idioma español, se desconoce si el instrumento posee las cualidades psicométricas para ser utilizada en la práctica en Puerto Rico. Ante esto, nace la interrogante de ¿cuáles son las propiedades psicométricas de la Escala de Autoeficacia en el Trabajo de Pepe et al. (2010) en una muestra de personas que trabajan en Puerto Rico?

Para contestar esta interrogante, este estudio propone analizar las propiedades psicométricas de dicha escala en una muestra de personas que trabajan en Puerto Rico. Esto, con el fin de poseer un instrumento con las propiedades psicométricas adecuadas y así aportar un conocimiento más amplio sobre la autoeficacia en el trabajo. A su vez, busca identificar vías de asesoramiento e intervención a nivel individual y organizacional, que contribuyan a contestar la siguiente pregunta: ¿podré ser eficaz en el trabajo?

\section{Autoeficacia en el Trabajo}

Bandura (1977) define el término de autoeficacia como las creencias de las propias capacidades para organizar y ejecutar los cursos de acción requeridos que producirán determinados logros o resultados. Asimismo, Bandura (1997) expone que la autoeficacia debe conceptualizarse de manera específica. La autoeficacia específica a un dominio hace referencia a la creencia de un individuo acerca de sus propias capacidades para el logro de un determinado resultado. Por lo tanto, la autoeficacia en el trabajo se define como la creencia de una persona de ser capaz de desempeñar bien el rol laboral (Cherniss, 1993). Maffei et al. (2012) definen el término como el conjunto de creencias que poseen los individuos de sus propias capacidades para llevar a cabo de manera exitosa las actividades asociadas al trabajo.

Según Bandura (1997), la autoeficacia se desarrolla a través de cuatro fuentes esenciales: el logro de desempeño o experiencia de dominio, el aprendizaje vicario, la persuasión social y el estado fisiológico o activación emocional. Su desarrollo es en función de cómo el individuo atiende y procesa cognitivamente la experiencia. El logro de desempeño involucra el dominio logrado en las tareas en el pasado y presente del trabajador. Martínez y Salanova (2006) indican que el éxito repetido en determinadas tareas aumenta las evaluaciones positivas de autoeficacia en el trabajo, mientras que los fracasos repetidos disminuyen la autoeficacia en el trabajo. De este modo, a la hora de asignar tareas a las personas que trabajan, se debe tener en cuenta su nivel de habilidad y competencia, para así ajustar las tareas según sus capacidades y no por debajo de sus competencias (Salanova et al., 2009).

La experiencia vicaria se desarrolla cuando la persona observa a otros ejecutar exitosamente ciertas actividades en el trabajo. Por consiguiente, la persona que trabaja puede llegar a creer que posee las capacidades suficientes para comportarse con igual éxito en su puesto de trabajo. Salanova et al. (2009) exponen que se debe tener en cuenta que algunas personas que trabajan no siempre tienen experiencia en la tarea a realizar, por lo que resulta muy útil fijarse en otras personas que trabajan que sí tienen esa experiencia y realizan la tarea de forma exitosa. Por lo tanto, pueden llegar a creer que también poseen las capacidades suficientes para comportarse con igual éxito.

La persuasión verbal, se refiere al refuerzo social, las críticas positivas, los halagos y las palabras de ánimo que se reciben por parte de los compañeros de trabajo. Según Salanova et al. (2009) la persona que trabaja y es persuadida verbalmente y además posee las capacidades de dominio necesarias para tener éxito en una determinada tarea, tiende a movilizar 
sus esfuerzos y mantenerlos, sin detenerse en sus deficiencias personales cuando surgen dificultades. Por consiguiente, la persuasión verbal como fuente de autoeficacia en el trabajo es limitada, pero puede ayudar a mejorar la eficacia, siempre y cuando la evaluación sea positiva dentro de límites realistas (Salanova et al., 2009).

Por último, las personas que trabajan también responden parcialmente a la información proporcionada por sus estados emocionales. La percepción que tengan de sus capacidades determinará qué tipo de actividades estarán dispuestos a elegir y cuán rápido aprenderán las tareas asignadas. Salanova et al. (2009) indican que las muestras de debilidad, como el nerviosismo o la sudoración, se pueden interpretar por parte de la persona que trabaja como falta de capacidad o competencia; mientras que las muestras positivas, como la tranquilidad, el buen ánimo o la alegría, son signos de dominio, competencia y control.

Con respecto a las fuentes de autoeficacia en el trabajo, Salanova et al. (2005) indican que el logro de ejecución es la fuente más importante de autoeficacia en el trabajo. Estos autores indican que el éxito de ejecución incrementa las evaluaciones positivas de autoeficacia en el trabajo, que a su vez influyen en el éxito futuro. Indican, además, que los fracasos repetidos aumentan las evaluaciones negativas de las propias competencias que a su vez incrementan las posibilidades de tener más fracasos en el futuro.

El constructo de autoeficacia en el trabajo se contextualiza dentro de la teoría social cognitiva (TSC) y la teoría de la autoeficacia de Bandura (1986). La TSC expone que las personas no son impulsadas por fuerzas internas, ni controladas y tampoco creadas automáticamente por estímulos externos. Esta teoría se basa en dos principios fundamentales: (1) la interacción recíproca entre factores personales, la conducta y el ambiente y (2) la existencia de las capacidades humanas que no se limitan al aprendizaje de habilidades y competencia. Posteriormente, Bandura establece una interacción dinámica entre factores, conocido como el determinismo recíproco, el cual consiste en: factores personales (cognitivos, afectivos y fisiológicos), el comportamiento observable del sujeto y, los factores situacionales. Estas interacciones producen una reciprocidad triádica.
Por otra parte, la teoría de la autoeficacia, postula que esta tiene un papel mediador dentro del sistema del determinismo recíproco (Bandura, 1986). Bandura expone que la autoeficacia se afecta por fuentes de información (cognitivas, conductuales y emocionales), y a su vez influye en la conducta y en los procesos cognitivos y emocionales. Según esta teoría, la autoeficacia se constituye por las expectativas de eficacia y de resultados. El mismo autor define las expectativas de eficacia como la creencia de un individuo al realizar satisfactoriamente la conducta requerida para producir unos resultados determinados, o la creencia de un individuo respecto a su capacidad para obtener un nivel específico de ejecución en un ambiente determinado. A su vez, las expectativas de resultados son las creencias del individuo respecto a las consecuencias o resultados que probablemente seguirán a una determinada acción. Por consiguiente, la creencia del individuo en una determinada conducta llevará a determinados resultados. La teoría expone que las expectativas de eficacia y de resultado pueden ocurrir simultáneamente en la mente del individuo al realizar determinadas conductas.

\section{¿Qué No es Autoeficacia en el Trabajo?}

Existen constructos que son muy similares a la autoeficacia en el trabajo, tales como la autoeficacia generalizada y el locus de control interno. Schwarzer (1999) indica que la autoeficacia generalizada se conceptualiza como la confianza del individuo en las propias destrezas de afrontamiento que se manifiestan en un rango amplio de situaciones retadoras y que poseen características estables (Schwarzer, 1999). Es decir, la autoeficacia generalizada es un constructo de la personalidad que genera habilidades y confianza en el individuo a la hora de afrontar un amplio espectro de situaciones (Pérez, 2015). Sin embargo, la autoeficacia específica en el trabajo se define como la creencia de las propias competencias del individuo a la hora de afrontar o resolver con éxito situaciones en el trabajo (Bandura,1997). La creencia de autoeficacia en el trabajo actúa como recurso frente a las demandas del entorno laboral favoreciendo el desempeño y la adaptación a los cambios. Asimismo, Bandura (2006) indica que la autoeficacia específica es el mejor predictor de la ejecución, a diferencia de la autoeficacia generalizada. Esto se debe, a que la autoeficacia específica puede explicar un amplio rango de conductas y resultados cuando el contexto es más específico. 
Por otro lado, el locus de control se define como el grado en que la personas se sienten responsables por los acontecimientos que le ocurren (Rotter, 1966); es un componente de la personalidad con rasgos únicos en el individuo (Vere-Noriega \& Cervantes-Mariscal, 2000). Existen dos tipos de locus de control: interno y externo. Cuando las situaciones se perciben como contingentes a la conducta del individuo, se refiere al locus de control interno (Rotter, 1966). En cambio, Rotter señala que, si la situación no es contingente con la conducta del individuo, se denomina locus de control externo. La autoeficacia en el trabajo, por el contrario, se refiere a la creencia que la persona posee sobre sus capacidades para ejecutar su trabajo (Bandura, 1997). Martínez y Salanova (2006) indican que la autoeficacia en el trabajo determina el locus de control interno; si un individuo se siente eficaz y cree poseer las habilidades necesarias en el trabajo, establecerá una relación entre sus acciones y los resultados. Por otra parte, según estas autoras, el locus de control determina la autoeficacia en el trabajo; si el individuo con locus de control interno no posee las habilidades necesarias parar realizar la tarea, es posible que el individuo no posea autoeficacia en el trabajo.

\section{Autoeficacia en el Trabajo y su Rol en las Organiza- ciones}

Es importante destacar el papel que desempeña la autoeficacia en el trabajo en las organizaciones. La autoeficacia en el trabajo es un constructo importante, ya que determina el modo de pensar, sentir, motivarse y actuar del individuo, por lo que contribuye de forma significativa en su trabajo (Martínez \& Salanova, 2006). La autoeficacia en el trabajo, indica la convicción de la persona que trabaja para cumplir un rol dentro de su entorno laboral (Calderón, 2018). En el ámbito laboral, los estudios demuestran que la autoeficacia en el trabajo es un importante predictor del desempeño y el rendimiento de las personas que trabajan en las organizaciones (Salanova et al., 2009). Asimismo, se comprobó que la autoeficacia incrementa positivamente el desempeño laboral a lo largo del tiempo (Salanova et al., 2009). Además, determina la calidad y adaptación de los individuos en su entorno laboral (Hernández \& Oramas, 2016). La autoeficacia en el trabajo juega un papel fundamental en las decisiones que el individuo toma sobre su trabajo, compromiso laboral, desempeño, persistencia y motivación (Ferrari et al., 2017). Del mismo modo, los estudios muestran que los individuos que poseen autoeficacia tienden a ser más optimistas y resilientes (LeBlanc et al., 2010). A su vez, la autoeficacia en el trabajo tiene un efecto de interacción con las demandas laborales (acoso laboral), de manera que los trabajadores con autoeficacia poseen conductas de afrontamientos en situaciones de estrés laboral (Meseguer et al., 2014). De igual manera, la autoeficacia en el trabajo genera un gran valor en los programas de formación, debido a que fomentan la creencia de eficacia, ayudan a la preparación, la intensidad y la satisfacción laboral (Martínez \& Salanova, 2006).

El poseer autoeficacia en el trabajo, determina el esfuerzo y la persistencia en la realización de las tareas, sobre todo en situaciones adversas (Pepe et al., 2010). Por otra parte, influye en los patrones de pensamientos y sentimientos de los individuos. Además, determina la cantidad de intento y tiempo que invierten para resolver obstáculos (Salanova et al., 2005). A su vez, la eficacia en el trabajo actúa como modulador entre los estresores, demanda laboral (sobrecarga de horas de trabajo, la rutina o la presión temporal) y en las conductas de afrontamiento ante el estrés laboral (Salanova et al., 2005). Del mismo modo, el poseer autoeficacia en el trabajo promueve la satisfacción laboral, fomenta el engagement (Spontón et al., 2018), promueve el compromiso organizacional y el rendimiento laboral (Walumbwa et al., 2011). Además, las personas que trabajan que poseen autoeficacia se encuentran orientadas hacia la innovación y promueven conductas emprendedoras (Lee et al., 2011),

Los niveles de autoeficacia en el trabajo influyen en el rendimiento y desarrollo de las competencias de las personas que trabajan (Navarro, 2016) y contribuyen positivamente en las condiciones del entorno laboral (Ramis et al., 2007). Las personas que trabajan con altos niveles de autoeficacia se caracterizan por su tenacidad y determinación, y por ser guiados por su creencia de éxito en el futuro. El poseer una elevada autoeficacia en el trabajo, favorece la dedicación de las personas que trabajan fomentando el aprendizaje e innovación (Lunenburg, 2011). Las personas que poseen altos niveles de autoeficacia enfrentan los retos a partir de la autopercepción, considerándose capaces, seguras y confiadas para superar diversos obstáculos o situaciones que se les presenten en el trabajo (Alvarino,2018). Además, aplican conductas de afrontamiento enfocadas en la solución del problema 
(Salanova et al., 2005). Estas personas que trabajan enfrentan efectivamente los retos y las demandas laborales, proveyéndole a la organización recursos humanos enérgicos, comprometidos con su labor y orgullosos de lo que hacen; más rentables para la organización, más centrados a sus tareas y con menos deseos de abandonar la organización (Hernández \& Oramas, 2016).

Por otra parte, las personas con niveles bajos de autoeficacia en el trabajo se enfocan más en sus emociones o en sí mismos (Alvarino, 2018). Los bajos niveles de autoeficacia en el trabajo se encuentran asociados a niveles elevados de burnout, depresión y ansiedad (Salanova et al., 2005). De igual forma, los niveles de eficacia en el trabajo afectan el rendimiento de las personas que trabajan (Alvarino, 2018). Niveles bajos de autoeficacia se manifiestan en niveles de desempeño bajo ante situaciones de estrés o circunstancias difíciles (Grau et al., 2012). Asimismo, la baja autoeficacia en el trabajo se asocia con pensamientos pesimistas, poco esfuerzo en la realización de tareas, bajas aspiraciones y compromiso laboral, enfoque en la autoevaluación y evitación de tareas difíciles (Consuelo, 2020). Las personas que se consideran poco eficaces en el trabajo poseen baja habilidad en la solución de problemas y en ocasiones pudieran aumentar sus niveles de deficiencia y dificultad del entorno laboral (Consuelo, 2020). Estos pensamientos negativos generan estrés y dificultan la utilización de los recursos disponibles. Es por esa razón, que el individuo debe adquirir un sentido de autoeficacia en el trabajo para llevar a cabo las tareas (Pepe et al., 2010).

\section{Medición de Autoeficacia en el Trabajo}

Al revisar la literatura existente se encontraron diferentes instrumentos para medir la autoeficacia en el trabajo. Avallon et al. (2007) desarrollaron el instrumento Work Self-Efficacy Scale (WSES) para medir la autoeficacia en el trabajo. El instrumento de 10 ítems tiene como objetivo evaluar las percepciones que tiene un individuo sobre sí mismo con respecto a los dominios de trabajo específico, tales como: la capacidad de manejar las relaciones interpersonales, trabajar con colegas con diferentes características y experiencias, comportarse de manera eficaz en el contexto laboral, aprender nuevos métodos de trabajo, respetar los horarios y plazos de trabajo, y lograr las metas establecidas. Posteriormente, Lagerveld et al. (2010) desarrollaron la escala Return to Work Self-Efficacy (RTW-SE) de 11 ítems para evaluar la percepción de los trabajadores sobre sus capacidades para realizar tareas habituales al regresar a la organización. Por su parte, Mazzetti et al. (2014) desarrollaron el instrumento Work Self-efficay Scale de 5 ítems para examinar la relación entre la adicción al trabajo, las características individuales y los factores ambientales de las personas que trabajan. Miraglia et al. (2015) desarrollaron Perceived Work Self-efficacy Scale de seis ítems con el propósito de examinar el rol de la autoeficacia y la tenacidad organizacional del desempeño laboral. De igual forma, Akkermans et al., (2015) elaboraron Work-Related Self-Efficacy Scale de cinco ítems con el objetivo de examinar la autoeficacia en el trabajo y su relación con los programas Career Skills. Del mismo modo, Fida et al. (2015) desarrollaron la escala Work Self-Efficacy Measure de 15 ítems para evaluar la autoeficacia en el trabajo con relación al desarrollo de los empleados y la autoeficacia emocional.

Tal y como se evidencia, no se han desarrollado escalas para medir la autoeficacia en el trabajo en el idioma español. Sin embargo, a través de la revisión de literatura se identificó un estudio donde se tradujo y validó la Escala de Autoeficacia en el Trabajo de Avallon et al. (2007) en una muestra en España e Italia (Pepe et al., 2010). La estructura de la escala indica dos factores: compromiso y voluntad racional. El primer factor se refiere a las percepciones de ser capaz de alcanzar los objetivos y comprometerse con el trabajo.

El segundo factor indica predisposición o relación con los compañeros y supervisores del trabajo. El instrumento está compuesto por 10 ítems con un anclaje de respuesta tipo Likert de cinco puntos que fluctúan entre 1 (nada bien) hasta 5 (muy bien). Pepe et al. realizaron un análisis de la estructura factorial y comprobación de la invarianza con el propósito de adaptar la escala Work Self-Efficacy Scale al idioma español. La muestra fue de 658 jóvenes de Italia y España entre las edades de 20 a 26 años. El modelo obtuvo un buen ajuste, los ítems mostraron carga factoriales de .60 con un rango de .61 a .82 en la muestra italiana y .61 a .76 en la muestra española. El coeficiente alfa de Cronbach para voluntad racional es de $\alpha=.85$ y para compromiso de $\alpha=.8$. 


\section{Propósito y Objetivos del Estudio}

Como se evidencia, hasta el momento no se han encontrado estudios que analicen las propiedades psicométricas de la Escala de Autoeficacia en el Trabajo (Pepe et al., 2010) en el contexto laboral en Puerto Rico. El objetivo de este trabajo es analizar las propiedades psicométricas de la Escala de Autoeficacia en el Trabajo de Pepe et al. (2010) en una muestra de personas trabajando en Puerto Rico. En términos específicos, este estudio tiene los siguientes objetivos:

1) Analizar la estructura factorial de la Escala de Autoeficacia en el Trabajo de Pepe et al. (2010) mediante el análisis de factores confirmatorio.

2) Analizar la capacidad de discriminación de los ítems de la escala.

3) Analizar la fiabilidad de la escala y sus factores mediante el índice de consistencia interna de alfa de Cronbach y la fiabilidad compuesta.

4) Analizar la validez convergente y discriminante mediante el análisis de varianza extraída distinguiendo la autoeficacia en el trabajo de la autoeficacia general y locus de control interno como variables de criterio.

\section{MÉTODO}

\section{Diseño de Investigación}

En el presente estudio se realizó un análisis secundario de datos de una investigación exploratoria (Martínez et al., 2019). El diseño utilizado en esta investigación fue no experimental transversal, de tipo instrumental (Montero \& León, 2007).

\section{Participantes}

Se llevó a cabo un análisis secundario de los datos obtenidos de una investigación que realizó Martínez et al (2019). La muestra de 678 personas trabajando se obtuvo por disponibilidad, de los cuales el $62 \%$ eran mujeres y $38 \%$ hombres. Las edades de los participantes oscilan entre 21 y 68 años $(M=39.5 ; D E=11.60)$. En cuanto a la preparación académica, el 39\% indicó poseer un grado de bachillerato. En relación con el número de años que llevan los participantes trabajando para su organización los mismos fluctúan entre 1 y 50 años $(M=14.74, D E=10.32)$. Respecto al tipo de organización donde trabajan, el $49 \%$ indicó trabajar para el sector privado. Por último, el 34\% indicó trabajar en el sector de la educación.

\section{Instrumentos}

Work Self-Efficacy Scale (WSES). Se utilizó la escala que Pepe et al. (2010) adaptaron al español e italiano. La escala en versión en español tiene 10 ítems y evalúa las creencias de eficacia sobre las actividades laborales. Tiene un formato de respuesta tipo Likert que va desde 1 (no del todo bien) a 5 (muy bien), permitiendo a los participantes evaluar qué tan capaces se sienten al llevar a cabo la acción o comportamiento en el trabajo. Los autores realizaron un análisis factorial confirmatorio donde dividen la escala en dos factores: compromiso y voluntad relacional. El factor de compromiso evalúa las percepciones de alcanzar objetivos fijos y comprometerse significativamente con su trabajo $(\alpha=.82)$. Por otra parte, el factor voluntad racional evalúa la predisposición o atención con sus compañeros y superiores $(\alpha=.85)$.

Escala de Locus de Control Interno. La escala que desarrollaron Suárez-Álvarez et al. (2016) evalúa el locus de control interno en diversos contextos (clínico, académico y laboral). Cuenta con 10 ítems, con un formato de respuesta de cinco puntos tipo Likert que comienzan desde 1 (totalmente desacuerdo) hasta 5 (totalmente de acuerdo). El instrumento cuenta con una confiabilidad alfa de Cronbach $\alpha=.87$.

Sub-escala de autoeficacia de OREA. La subescala de autoeficacia forma parte del Cuestionario de Optimismo, Resiliencia, Esperanza y Autoeficacia (OREA) que desarrollaron Meseguer-de Pedro et al. (2017). El instrumento evalúa el capital psicológico positivo en el ámbito laboral. Esta subescala está formada por tres ítems que se responden en una Escala Likert donde 1 significa (totalmente incierto) a 4 (totalmente cierto). La subescala de autoeficacia cuenta con un alfa de Cronbach de fiabilidad del $\alpha=.82$.

\section{Procedimientos Generales}

En este estudio se utilizaron datos secundarios provenientes de la investigación que realizaron Martínez et al. (2019). Para propósitos de nuestro estudio, se obtuvo el permiso de los investigadores para utilizar los datos recopilados. Luego de obtener el permiso de los investigadores, estos fueron compilados en los programas Stata 16, IBM SPSS Statistics versión 25 y $R$.

El propósito de esta investigación fue analizar las propiedades psicométricas de la Escala de 
Autoeficacia en el Trabajo. Con eso en mente, en primer lugar, se realizó un análisis de normalidad multivariante y se corrigieron los índices de ajuste mediante las correcciones de Satorra y Bentler (2001). Luego de completar el análisis factorial de la escala se realizó un análisis de discriminación de ítems utilizando la técnica de correlación ítem-total. Además, se calculó la consistencia interna de la escala utilizando el coeficiente alfa de Cronbach. Por último, se procedió a examinar la validez convergente y discriminante mediante la varianza media extraída, como exponen Fornell y Larcker (1981). Se analizó la validez discriminante de la Escala de Autoeficacia en el Trabajo (Pepe et al., 2010) con la Escala de Locus de Control Interno (Suárez-Álvarez et al., 2016) y la Sub-escala de autoeficacia de OREA (Meseguer-de Pedro et al., 2017).

\section{RESULTADOS}

\section{Análisis Descriptivos de los Ítems}

Se calcularon las medias y desviaciones estándar para los 10 ítems de la Escala de Autoeficacia en el trabajo de Pepe et al. (2010) para analizar las propiedades de distribución. Las medias de los ítems fluctuaron entre 3.48 y 3.67, mientras que las desviaciones estándar fluctuaron entre .48 y .63. Al calcular los estadísticos de normalidad de Kolmogorov-Smirnov y Shapiro-Wilk se evidenció que los datos no siguen una distribución normal $(p<.001)$. Asimismo, se analizó la normalidad multivariante de los datos utilizando las pruebas estadísticas $M$ de Mardia, DoornikHansen y Henze-Zirkler (Doornik \& Hansen, 2008). Los resultados muestran que no hay normalidad multivariante de los datos: $M$ de asimetría $=37.35571, X 2(220)$ $=4224.604, p<.001 ; M$ de curtosis $=275.3142, X 2(1)=$ 16961.126, $p<.001$; Henze-Zirkler $=99.96129, X 2(1)=$ 3.04, $p<.001$; Doornik-Hansen X2(20) $=1243.732$, $p<$ .001. Por consiguiente, al no cumplirse con la normalidad de los datos se utilizaron las correcciones de Satorra y Bentler (2001) para calcular el ajuste de los modelos de ecuaciones estructurales.

\section{Análisis Factorial de la Escala}

En esta investigación se analizó la estructura factorial de la Escala de Autoeficacia en el Trabajo de Pepe et al. (2010) mediante el análisis confirmatorio de factores con ecuaciones estructurales con el método de estimación de máxima verosimilitud. En este proceso se pusieron a prueba dos modelos. El primero fue un modelo base (M1) en donde los 10 ítems de la escala representaban un solo factor latente. Los resultados del análisis confirmatorio para el M1 no presentaron un buen ajuste para los datos (véase Tabla 1). Ante esto, se procedió a poner a prueba un modelo de dos factores con 5 ítems por factor (M2) como se propone en la escala. Los primeros cinco ítems representaban el factor compromiso y los ítems restantes representaban el factor de voluntad relacional. Los resultados del análisis de factores demostraron que el M2 posee un buen ajuste para los datos (ver Tabla 1). El análisis confirmatorio de dos factores demostró poser un buen ajuste a los datos, $\chi 2=179.034$ (34) $p<.001$, RMSEA $=.08, S R M R=.04, C D=.97, C F I=.96, T L I=$ .95, $A I C=221.03 ;$ AIC corregido $=180.44 ; \chi 2$ corregido $=70.65(34) p<.001$, RMSEA corregido = .039; CFI corregido $=.98, T L I$ corregido $=.97$. Estos índices de ajuste relativos cumplen con los niveles aceptables propuestos por Satorra y Bentler (2001) (ver figura 1).

Por otra parte, Schumacker y Lomax (2010) recomiendan utilizar el AIC para comparar modelos de ecuaciones estructurales cuando los valores de $\chi^{2}$ son estadísticamente significativos. Ante esto, M2 presenta un índice menor $($ AIC $=6772.019)$ que los obtenidos por el primer modelo. Esto es indicativo de que el M2 tiene un mejor ajuste para los datos.

\section{Análisis de los Ítems}

Se examinó la capacidad de discriminación de los 10 ítems mediante el índice de correlación ítem total. Los mismos oscilaron entre .63 y .75, mientras que la varianza explicada en los ítems oscila entre .45 y .55 (véase Tabla 2). Los índices de discriminación que se encontraron están por encima del mínimo recomendado de .30 (Kline, 2005).

\section{Análisis de Fiabilidad}

Se analizó la confiabilidad y la fiabilidad compuesta. Los valores Alfa de Cronbach fueron los siguientes: para el factor compromiso .86 y para el factor en voluntad .88. En cuanto a la fiabilidad compuesta los valores son: compromiso .88 y voluntad .89. En la Tabla 3 se presentan los valores de fiabilidad compuesta para cada constructo. 


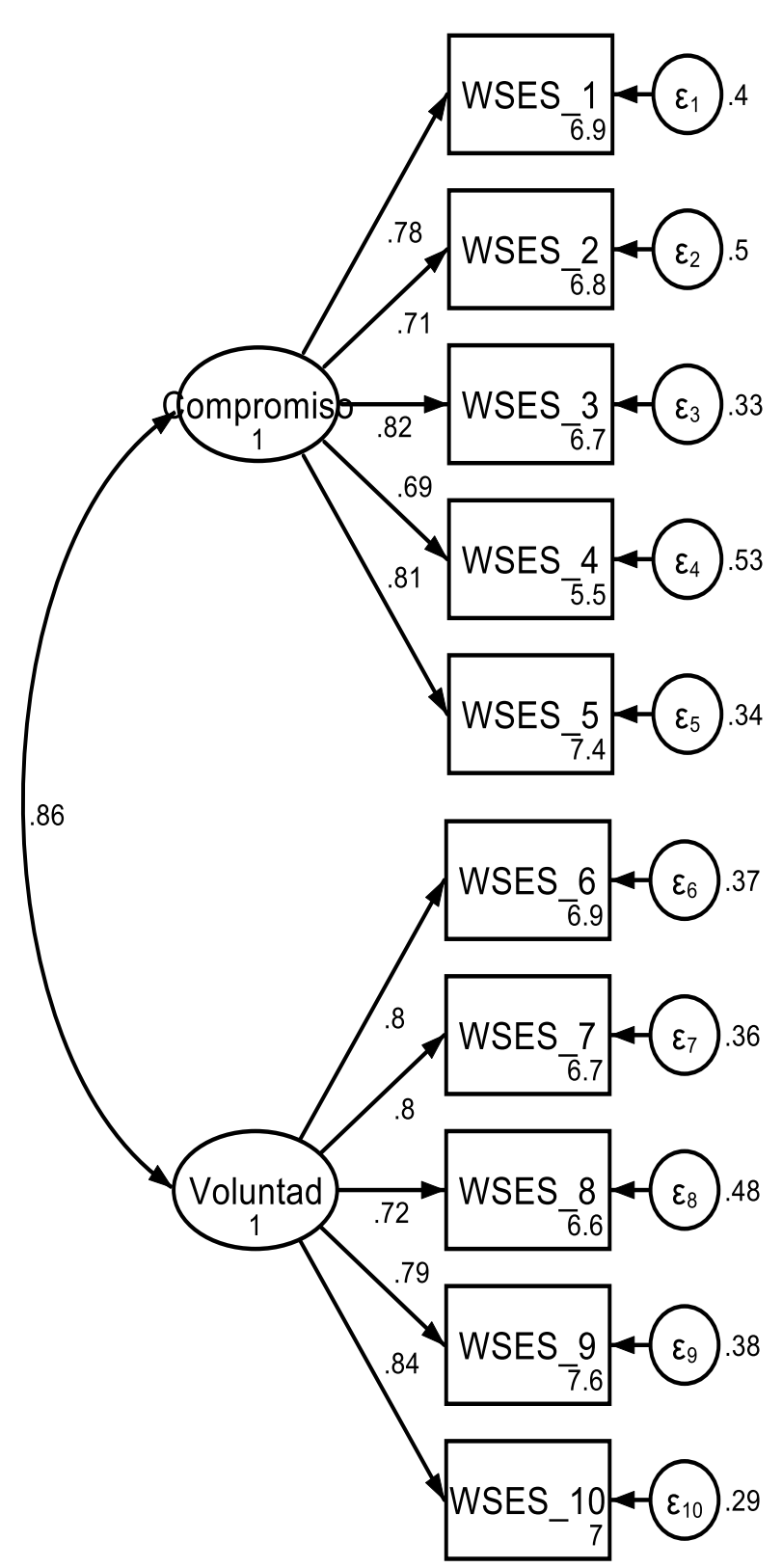

Figura 1. Modelo de dos factores de la Escala de Autoeficacia en el Trabajo de Pepe et al. (2010).

\section{Análisis de Validez Convergente y Discriminante}

Se analizó la validez convergente y discriminante mediante la Varianza Media Extraída (VME), la cual mide el promedio de varianza explicada por el constructo en los ítems. Valores altos en el VME indican que la varianza de error es menor. Los valores de VME de todos los constructos que se analizaron son: compromiso .58, voluntad .63, locus de control .47 y autoeficacia .52 (véase Tabla 3). Fornell y Larcker

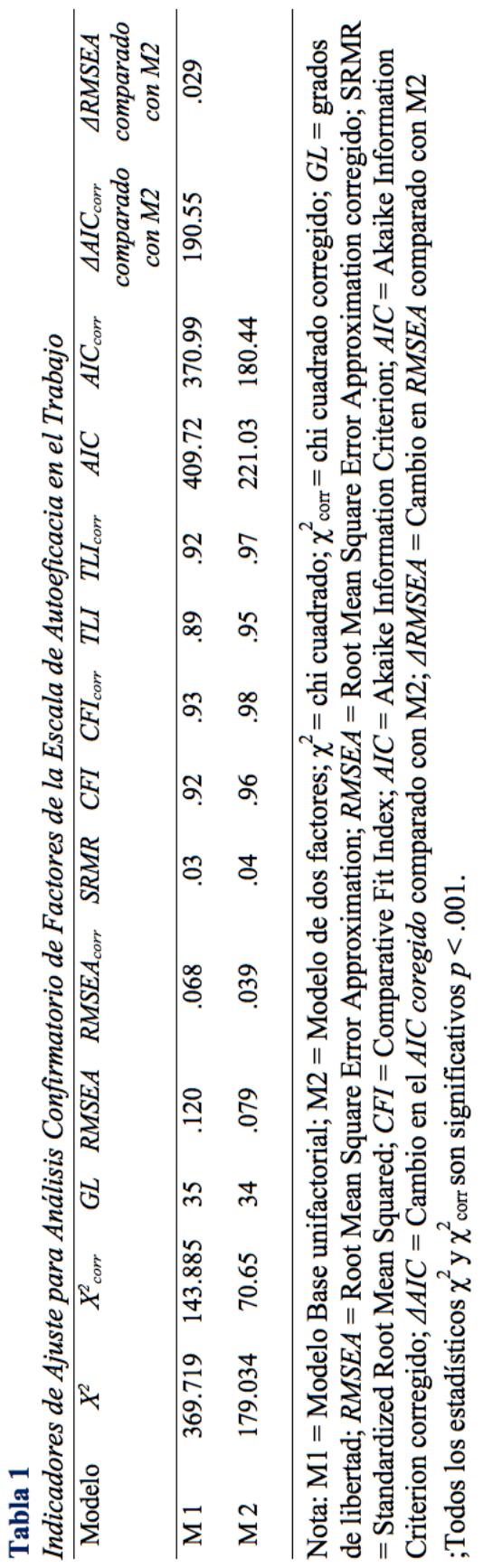

(1981) consideran que estos valores deben ser igual o mayor de .50 para ser considerados aceptables. Por consiguiente, el valor que obtuvo el locus de control interno no cumple con tener una VME igual o mayor a .50, por lo que su análisis debe ser tomado con precaución. Además, se procedió a examinar la relación entre los constructos mediante la correlación de $r$ de Pearson. Las correlaciones entre los factores fluctuaron entre .35 y .76 (ver tabla 3 ). Por otra parte, Fornell y Larcker (1981) indican que la varianza compartida 
entre dos constructos debe ser menor a la varianza explicada por cada uno de los constructos, haciendo que se cumpla con el criterio de validez discriminante. Lo que indica, que la Escala de Autoeficacia en el Trabajo no cumple con el criterio de validez discriminante cuando se administra a este grupo de participantes en Puerto Rico.

\section{Tabla 2}

Índices de Discriminación y Varianza Explicada de los Ítems de la Escala de Autoeficacia en el Trabajo

\begin{tabular}{|c|c|c|c|}
\hline \multicolumn{2}{|r|}{ Ítems } & \multirow{2}{*}{$\frac{r b i s}{.70}$} & \multirow{2}{*}{$\begin{array}{l}R^{2} \\
.55\end{array}$} \\
\hline 1. & $\begin{array}{l}\text { Lograr los objetivos que me } \\
\text { asignen. }\end{array}$ & & \\
\hline 2. & $\begin{array}{l}\text { Respetar los horarios y los } \\
\text { tiempos de trabajo }\end{array}$ & .65 & .47 \\
\hline 3. & $\begin{array}{l}\text { Aprender nuevos métodos de } \\
\text { trabajo. }\end{array}$ & .74 & .59 \\
\hline 4. & $\begin{array}{l}\text { Concretar todas mis energías } \\
\text { en el trabajo. }\end{array}$ & .63 & .45 \\
\hline 5. & Terminar el trabajo asignado. & .75 & .61 \\
\hline 6. & $\begin{array}{l}\text { Colaborar con los otros com- } \\
\text { pañeros }\end{array}$ & .75 & .59 \\
\hline 7. & $\begin{array}{l}\text { Trabajar con personas de edad } \\
\text { y experiencias que difieren a } \\
\text { las mías. }\end{array}$ & .72 & .60 \\
\hline 8. & $\begin{array}{l}\text { Tener buenas relaciones con } \\
\text { los jefes. }\end{array}$ & .68 & .50 \\
\hline 9. & $\begin{array}{l}\text { Relacionarme de manera efi- } \\
\text { caz con los clientes. }\end{array}$ & .72 & .58 \\
\hline 10. & Trabajar en equipo. & .75 & .65 \\
\hline
\end{tabular}

Nota. $R^{2}=$ Varianza explicada; $r b i s=$ Índice de discriminación; Ítems del 1 al 5 = Compromiso; Ítems del 6 al 10 = Voluntad.

Tabla 3

Medias, Desviaciones Estándar, Alfas, Fiabilidad Compuesta, Varianza Media Extraída y Correlaciones $(n=678)$

\begin{tabular}{lccccccccc}
\hline & $M$ & $D E$ & $a$ & $F C$ & $V M E$ & 1 & 2 & 3 & 4 \\
\hline 1. Compromiso & 3.64 & 2.19 & .88 & .88 & .58 & - & .86 & .54 & .51 \\
2. Voluntad & 3.60 & 2.20 & .86 & .89 & .63 & .76 & - & .50 & .42 \\
3. LCI & 3.50 & 4.73 & .88 & .90 & .47 & .43 & .46 & - & .50 \\
4. Autoeficacia & 3.55 & 1.37 & .76 & .76 & .52 & .35 & .42 & .41 & - \\
\hline
\end{tabular}

Nota. $L C I=$ Locus de Control Interno $M=$ media; $D E=$ desviación estándar; $a=$ alfa de Cronbach; $F C=$ fiabilidad compuesta; $V M E=$ varianza media extraída. Todas las correlaciones fueron significativas a $p<.001$. Los valores sobre la diagonal representan las correlaciones entre los factores latentes, mientras que los valores por debajo de la diagonal representan las correlaciones de las puntuaciones directas.

\section{DISCUSIÓN}

El objetivo de este estudio fue examinar las propiedades psicométricas de la Escala de Autoeficacia en el Trabajo de Pepe et al. (2010) en una muestra de personas empleadas en Puerto Rico. Los resultados indican que la Escala de Autoeficacia en el Trabajo, cuando se administra a un grupo de participantes en Puerto Rico, no posee índices adecuados respecto a su validez discriminante para poder utilizarse en investigaciones y diagnóstico organizacional como medida de autoeficacia en el trabajo. Los resultados de esta investigación demuestran que la escala no cumple con los criterios de validez discriminante entre los factores de voluntad y compromiso. Las correlaciones entre los factores son altas, lo que indica que existe mucha varianza compartida entre los factores. Es decir, el factor latente de voluntad explica la misma cantidad de varianza en los ítems de compromiso, tal y como lo hace el factor latente de compromiso en sus ítems.

Para abundar más, se realizó un análisis cualitativo de los ítems y se encontró que existe redundancia entre los factores que componen la escala. Por ejemplo, algunos ítems del factor voluntad (Colaborar con los otros compañeros; Trabajar con personas de edad y experiencias diferentes a las mías) pudieran estar midiendo el factor de compromiso y el de voluntad a la misma vez. Al analizar las definiciones de los factores, los autores de la escala entienden por voluntad, la predisposición o relación con los compañeros y supervisores del trabajo. Respecto al compromiso, este se refiere a las percepciones de ser capaz de alcanzar los objetivos y comprometerse con el trabajo. Basándonos en estas definiciones, el colaborar con los companeros pudiera medir tanto el factor compromiso como el factor voluntad, debido a que la colaboración entre los compañeros es fundamental para alcanzar las metas y los objetivos de la organización. Asimismo, los empleados deben ser capaces de trabajar con personas de edad y experiencias diferentes para alcanzar los objetivos de la organización. Como se observa, estos ítems pudieran estar midiendo ambos factores a la misma vez.

Otra de las razones por las cuales entendemos que la escala no posee las propiedades psicométricas deseadas radica en los planteamientos que presenta Bandura (2006). Este expone en su artículo Guide for 
Constructing Self-efficacy Scales que los ítems que compongan las escalas o instrumentos para medir autoeficacia deben reflejar con precisión el constructo. Bandura señala que los ítems deben redactarse en términos de puedo hacer en lugar de lo haré. Asimismo, expone que la autoeficacia debe medirse empleando niveles de demandas de tareas que representan diferentes grados de desafío o impedimento para un rendimiento exitoso. Los instrumentos de autoeficacia evalúan los niveles de dificultad que los individuos creen que pueden superar de su entorno. Es decir, si en los ítems del instrumento de autoeficacia, no se presentan los obstáculos que se deben enfrentar, se pudiera pensar que todos los individuos poseen niveles altos de autoeficacia. Al analizar de forma cualitativa los ítems de la escala, se observa que estos no siguen las guías de construcción de escalas de autoeficacia que estableció Bandura. Por consiguiente, esto podría ser un factor que influye en la validez discriminante $y$, a su vez, en las propiedades psicométricas de la escala. Es por tal razón, que realizamos una adaptación de los ítems de la escala cumpliendo con las recomendaciones que estableció Bandura (véase Tabla 4).

Cabe destacar que Pepe et al. (2010) analizaron la estructura factorial de la escala en España e Italia y los resultados del análisis indican que la escala posee validez. Es decir, la escala cumple con los criterios adecuados de validez en una población de España e
Italia. Sin embargo, los resultados de nuestra investigación no son comparables con los obtenidos por Pepe et al. Por consiguiente, estos resultados nos llevan a cuestionar la medición del constructo de la autoeficacia en el trabajo en Puerto Rico y Latinoamérica con la escala tal en su versión actual. Esto, debido a que es de suma importancia que los instrumentos posean índices adecuados de validez para así poderse utilizar en investigaciones y diagnósticos organizacionales.

Estos resultados representan un importante aporte para el desarrollo de nuevas investigaciones sobre la medición del constructo de autoeficacia en Puerto Rico y Latinoamérica. A su vez, permitirá un análisis más amplio sobre la investigación y construcción de las escalas de autoeficacia específica a un dominio. Esto, con el fin de aportar y evidenciar lo expuesto por Bandura (2006) relacionado a que la autoeficacia debe medirse de manera específica y que los ítems deben representar diferentes grados de desafíos o impedimentos para un rendimiento exitoso. La evaluación de la autoeficacia surge de los niveles de dificultad que los individuos pueden sobrellevar.

\section{Fortalezas y Limitaciones}

Como toda investigación, el presente estudio posee algunas limitaciones y fortalezas. En primer lugar, el muestreo del estudio original se obtuvo por

Tabla 4

Adaptación de la Escala de Autoeficacia en el Trabajo

\begin{tabular}{|c|c|}
\hline Ítems de la escala original & Ítems adaptados con obstáculos \\
\hline $\begin{array}{l}\text { 1. Lograr los objetivos que me asig- } \\
\text { nen. }\end{array}$ & 1. Lograr los objetivos que me asignen, aunque encuentre muchos obstáculos. \\
\hline $\begin{array}{l}\text { 2. Respetar los horarios y los tiempos } \\
\text { de trabajo. }\end{array}$ & $\begin{array}{l}\text { 2. Respetar los horarios y los tiempos de trabajo, aunque tenga que resolver si- } \\
\text { tuaciones inesperadas }\end{array}$ \\
\hline $\begin{array}{l}\text { 3. Aprender nuevos métodos de tra- } \\
\text { bajo. }\end{array}$ & $\begin{array}{l}\text { 3. Aprender nuevos métodos de trabajo, aunque dedique mucho tiempo y ener- } \\
\text { gía. }\end{array}$ \\
\hline $\begin{array}{l}\text { 4. Concretar todas mis energías en el } \\
\text { trabajo. }\end{array}$ & $\begin{array}{l}\text { 4. Concretar todas mis energías en el trabajo, aunque tenga que resolver situacio- } \\
\text { nes difíciles. }\end{array}$ \\
\hline 5. Terminar el trabajo asignado. & 5. Terminar el trabajo asignado, aunque me encuentre en una situación difícil. \\
\hline $\begin{array}{l}\text { 6. Colaborar con los otros compañe- } \\
\text { ros. }\end{array}$ & 6. Colaborar con los otros compañeros, aunque algunos se opongan. \\
\hline $\begin{array}{l}\text { 7. Trabajar con personas de edad y ex- } \\
\text { periencias que difieren a las mías. }\end{array}$ & $\begin{array}{l}\text { 7. Trabajar con personas de edad y experiencias que difieren a las mías, aunque } \\
\text { tengamos pensamientos distintos. }\end{array}$ \\
\hline $\begin{array}{l}\text { 8. Tener buenas relaciones con los je- } \\
\text { fes. }\end{array}$ & 8. Tener buenas relaciones con los jefes, aunque aparezcan situaciones difíciles. \\
\hline $\begin{array}{l}\text { 9. Relacionarme de manera eficaz con } \\
\text { los clientes. }\end{array}$ & $\begin{array}{l}\text { 9. Relacionarme de manera eficaz con los clientes, aunque se presenten aconteci- } \\
\text { mientos inesperados. }\end{array}$ \\
\hline 10. Trabajar en equipo. & 10. Trabajar en equipo, aunque sucedan situaciones imprevistas. \\
\hline
\end{tabular}


disponibilidad, por lo que no se pueden generalizar sus resultados a la población de Puerto Rico. Sin embargo, aunque fue por disponibilidad, la muestra de 678 participantes es heterogénea y facilitó la recopilación de información en diversos grupos demográficos y organizacionales. A pesar de las limitaciones que se identificaron, este estudio llevó a cabo un análisis exhaustivo cuantitativo y cualitativo de la Escala de Autoeficacia en el Trabajo. Por consiguiente, para futuras investigaciones se recomienda realizar una adaptación y validación de la escala modificada con los obstáculos para comprobar las propiedades psicométricas de la escala en Puerto Rico y Latinoamérica.

\section{CONCLUSIÓN}

Actualmente el trabajo es una actividad fundamental en la vida de cada ser humano. Sin embargo, en los últimos años observamos una creciente preocupación por las condiciones laborales de las personas en Puerto Rico y en otros países. Por consiguiente, las organizaciones necesitan instrumentos que estén adaptados a la cultura. Es por esta razón, que es de suma importancia analizar las propiedades psicométricas de la escala propuesta en este estudio. En este sentido, esta investigación ofrece la posibilidad de poseer en el futuro y, siguiendo las recomendaciones que se realizaron, un instrumento con las propiedades psicométricas adecuadas para utilizarse tanto en el ámbito investigativo como en el de la práctica profesional en contextos laborales puertorriqueños. Por lo tanto, aún queda una preguntar sin contestar: ¿Podré ser eficaz en el trabajo?

$\mathrm{Si}$ bien es cierto que este estudio representa un paso de avance en la medición del constructo de la autoeficacia en el trabajo en Puerto Rico, también abre paso para realizar un análisis psicométrico de la Escala de Autoeficacia en el Trabajo con los ítems propuestos en este estudio. Es nuestro interés que las futuras investigaciones puedan explorar y desarrollar instrumentos de medición que se adapten a la cultura laboral en Puerto Rico.

Financiamiento: La presente investigación no fue financiada por alguna entidad ni patrocinador.

Conflicto de Intereses: No existen conflictos de intereses de parte de los autores de la investigación.

Aprobación de la Junta Institucional para la Protección de Seres Humano en la Investigación: Universidad Carlos Albizu.
Consentimiento o Asentimiento Informado: Todo participante completó un consentimiento informado.

Proceso de Revisión: Este estudio ha sido revisado por pares externos en modalidad de doble ciego.

\section{REFERENCIAS}

Akkermans, J., Brenninkmeijer, V., Schaufeli, W. B., \& Blonk, R. W. B. (2015). It's all about career skills: Effectiveness of a career development intervention for young employees. Human Resource Management, 54(4), 533-551. https://doi.org/10.1002/hrm.21633

Alvarino, J. (2018). Niveles de autoeficacia laboral en profesionales de los establecimientos de salud de la cuidad de Cajamarca (Disertación doctoral, Universidad Privada Antonio Guillermo Urrelo). Repositorio Intitucional- La Universidad de Cajamarca.

Avallone, F., Pepe, S., \& Porcelli, R. (2007). Autoefficacia percepita nella ricerca del lavoro: scale di misura. In Isfol, Bisogni, valori e autoefficacia nella scelta del lavoro. ISFOL, 133-142.

Bandura, A. (1977). Social learning theory. Editorial Prentice-Hall.

Bandura, A. (1986). Social foundations of thought and action: A social cognitive theory. Editorial Prentice-Hall. https://doi.org/10.1037/0003-066X.44.9.1175

Bandura, A. (1997). Self-efficacy: The exercise of control. Editorial Henry Holt \& Co.

Bandura, A. (1999). A social-cognitive theory of personality. In L. Pervin \& O. John (Eds.), Handbook of personality, (pp. 154 -196). Editorial Guilford Press.

Bandura, A. (2001). Social cognitive theory: an agentic perspective. Annual Review of Psychology, 52, 1-26. https://doi.org/10.1146/annurev.psych.52.1.1

Bandura, A. (2006) Guide for constructing self-efficacy scales. En F. Pajares \& T. Urdan (Eds.). Self-efficacy beliefs of adolescents, age information publishing (pp. 307-337). Editorial Greenwich.

Calderón, M. (2018). Relación de la satisfacción laboral afectiva y la autoeficacia laboral en trabajadores profesionales peruanos (Disertación doctoral, Universidad San Ignacio de Layola). Repositorio Institutional- Universidad San Ingnacio de Layola.

Consuelo, N. (2020). Autoeficacia laboral y satisfacción laboral en trabajadores de recursos humanos de una consultora privada de Lima Metropolitana (Disertación doctoral, Universidad San Ignacio de Loyola). Repositorio Institucional- Universidad San Ignacio de Layola.

Cherniss, C. (1993). Role of professional self-efficacy in the etiology and amelioration of burnout. En W. Schaufeli \& C. Maslach (Eds.). Professional burnout: Recent developments in theory and research (pp. 135-150). Editorial Taylor-Francis.

Doornik, J. A., \& Hansen, H. (2008). An omnibus test for univariate and multivariate normality. Oxford Bulletin of Economics and Statistics, 70, 927-939. https://doi.org/10.1111/j.14680084.2008.00537.x

Ferrari, H., Nunes M., \& Marín, F. (2017). Autoeficacia en el trabajo: Revisión bibliométrica entre 2004 y 2014 en la base de datos 
ebsco-Academic Search. Psicología desde el Caribe, 3(3), 204-218. http://dx.doi.org/10.14482/psdc.34.3.11141

Fida, R., Paciello, M., Tramontano, C., Barbaranelli, C., \& Farnese, ML (2015). "Yes, I Can": The protective role of personal selfefficacy in hindering counterproductive work behavior under stressful conditions. Anxiety, Stress \& Coping: An International Journal, 28(5), 479-499. https://dx.doi.org/10.1080/10615806.2014.969718

Fornell, C., \& Larcker, D. F. (1981). Evaluating structural equation models with unobservable variables and measurement error. Journal of Marketing Research, 18(2), 39-50. https://doi.org/10.2307/3151312

Grau, R., Salanova, M., \& Peiró, J.M. (2000). Efectos moduladores de autoeficacia en el estrés laboral. Apuntes de Psicología, 18, 5775.

http://www.apuntesdepsicologia.es/index.php/revista/article/ view/414/334

Hernández, L., \& Oramas, A. (2016). Autoeficacia y work engagement en trabajadores de diferentes ocupaciones asistenciales. Revista Cubana de Salud y Trabajo, 17(4), 232-41. http://www.revsaludtrabajo.sld.cu/index.php/revsyt/article/vi ew/42/54

Kline, T. J. B. (2005). Psychological testing: A practical approach to design and evaluation. Editorial SAGE Publications.

Lagerveld, E., Blonk, W., Brenninkmeijer, V., \& Schaufeli, W. (2010). Return to work among employees with mental health problems: Development and validation of a self-efficacy questionnaire. Work \& Stress, 24(4), 359-375. https://dx.doi.org/10.1080/02678373.2010.532644

LeBlanc, P., Schaufeli, W.B., Salanova, M., Llorens, S., \& Nap, R.E. (2010). Efficacy beliefs predict collaborative practice among intensive care unit nurses. Journal of Advanced Nursing, 3, 583-594. https://dx.doi.org/10.1111/j.1365-2648.2009.05229.x

Lee, L., Wong, P. K., Der Foo, M., \& Leung, A. (2011). Entrepreneurial intentions: The influence of organizational and individual factors. Journal of business venturing, 26(1), 124-136. https://doi.org/10.1016/j.jbusvent.2009.04.003

Lunenburg, F. C. (2011). Self-efficacy in the workplace: Implications for motivation and performance. International Journal of management, business, and administration, 14(1), 1-6. https://doi.org/10.5539/ijbm.v8n14p80

Maffei, L., Spontón, C., Spontón, M., Castellano, E., \& Medrano, L. (2012). Adaptación del Cuestionario de Autoeficacia Profesional (AU-10) a la población de trabajadores cordobeses. Pensamiento Psicológico, 10 (1), 51-62. http://revistas.javerianacali.edu.co/index.php/pensamientopsicologico/article/view/178

Martínez, I. (2004). Autoeficacia aplicada al trabajo y a las organizaciones. En M. Salanova, R. Grau, I. Martínez, E. Cifre, S. Llorens, \& M. García-Renedo (Eds.). Nuevos horizontes en la investigación sobre autoeficacia (pp.178-186). Editorial Publicaciones de la Universitat Jaume I.

Martínez, I., \& Salanova, M. (2006). Autoeficacia en el trabajo: el poder de creer que tú puedes. Estudios Financieros, 279, 175-202. http://www.want.uji.es/download/autoeficacia-en-el-trabajoel-poder-de-creer-que-tu-puedes/

Martínez, M., Peña, O., Blanco, S., Román, Y., Cabrera, B., Figueroa, W., López, S., \& Pérez, M. (2019). Capital psicológico y su relación con variables individuales y organizacionales. Manuscrito no publicado, Universidad Carlos Albizu, Puerto Rico.

Mazzetti, G., Schaufeli, WB y Guglielmi, D. (2014). Are workaholics born or made? Relations of workaholism with person characteristic and overwork climate. International Journal of Stress Management, 21(3), 227-254. https://doi.org/10.1037/a0035700

Meseguer-de Pedro, M., Soler-Sánchez, M., Fernández-Valera, M., \& García-Izquierdo, M. (2017). Evaluación del capital psicológico en trabajadores españoles: Diseño y estructura empírica del cuestionario OREA. Anales de Psicología, 33(3), 714-721. https://doi.org/10.6018/analesps.33.3.261741

Meseguer, M., Soler, M., \& García-Izquierdo, M. (2014). El papel moderador de la autoeficacia profesional entre situaciones de trabajo laboral y la salud en una muestra multiocupacional. Anales de Psicología, 30 (2), 573-578 https://doi.org/10.6018/analesps.30.2.161251

Miraglia, M., Alessandri, G., \& Borgogni, L. (2015). Trajectory classes of job performance. Career Development International, 20(4). 424-442. http://dx.doi.org/10.1108/CDI-03-2015-0032

Montero, I., \& León, O. G. (2007). A guide for naming research studies in psychology. International Journal of Clinical and Health Psychology, 7(3), 847-862.

Navarro, L. (2016). Fundamentos teóricos de la autoeficacia. En L. Prieto (Eds.), Autoeficacia el profesor universitario: eficacia percibida y practica docente (pp.65-109). Editorial Naceas.

Pepe, S., Farnese, M. L., Avallone, F., \& Vecchione, M. (2010). Work Self-efficacy Scale and Search for Work Self-efficacy Scale: A validation study in Spanish and Italian cultural contexts. Revista de Psicología del Trabajo y de las Organizaciones, 26, 201210. https://doi.org/10.5093/tr2010v26n3a4

Pérez, C. (2015). Inserción laboral de universitarios desde la perspectiva psicosocial (Disertación doctoral, Universidad de Extremadura). Repositorio Institucional- Universidad Extremadura.

Ramis, M., Manassero, M., Ferrer, V., \& García, E. (2007). Influencia de las habilidades comunicativas de la dirección sobre la motivación, la autoeficacia y laa satisfación de sus equipos de trabajo. Revista de Psicología del Trabajo y de las Organizaciones, 23(2), 161-181. https://www.redalyc.org/articulo.oa?id=231317597001

Raykov, T., \& Shrout, P. E. (2002). Reliability of scales with general structure: Point and interval estimation using a structural equation modeling approach. Structural equation modeling, 9(2), 195-212. https://doi.org/10.1207/S15328007SEM0902_3

Rotter, J. B. (1966). Generalized expectancies for internal versus external control of reinforcement. Psychological Monographs: General and Applied, 80, 1-28. https://doi.org/10.1037/h0092976

Salanova, M., Grau, RM., \& Martínez, IM. (2005). Demandas laborales y conductas de afrontamiento: El rol modulador de la autoeficacia profesional. Psicothema, 17 (3), 390-395. http://www.psicothema.com/pdf/3118.pdf 
Salanova, M., Lorente, L., \& Vera, M. (2009). Recursos personales: las creencias de eficacia. En M. Salanova (Eds.), Psicología de la salud ocupacional (pp. 49-171). Editorial Síntesis.

Satorra, A., \& Bentler, P. M. (2001). A scaled difference chi-square test statistic for moment structure analysis. Psychometrika, 66(4), 507-514. https://doi.org/10.1007/BF02296192

Schumacker, R. E., \& Lomax, R. G. (2010). A beginner's guide to structural equation modeling (3rd Ed.). Editorial Mahwah.

Schwarzer, R. (1999). General perceived self- efficacy in 14 cultures. Self-efficacy assessment. Academic Website: www.yorku.ca/faculty/academic/schwarze/world14.htm

Spontón, C., Castellano, E., Salanova, M., Llorens, S., Maffei, L., \& Medrano, L. (2018). Evaluación de un modelo sociocognitivo de autoeficacia, burnout y engagement en el trabajo: análisis de invarianza entre Argentina y España. Psychology, 12(1), 89-101. https://doi.org/10.21500/19002386.3226

Suárez-Álvarez, J., Pedrosa, I., García-Cueto, E., \& Muñiz, J. (2016). Locus of control revisited: Development of a new bi-dimensional measure. Anales de Psicología, 32(2), 578-586. https://doi.org/10.6018/analespd.32.2.20078

Vera Noriega, J. A., \& Cervantes Mariscal, N. E. (2000). Locus de control en una muestra de residentes del noroeste de México. $\begin{array}{llll}\text { Psicología } \quad \text { y } & \text { Salud, } & \text { 237-247. }\end{array}$ https://www.researchgate.net/publication/289378187_Locus_de_c ontrol_en_una_muestra_de_residentes_del_Noroeste_de_Mexico

Walumbwa, F. O., Mayer, D. M., Wang, P., Wang, H., Workman, K., \& Christensen, A. L. (2011). Linking ethical leadership to employee performance: The roles of leader member exchange, self-efficacy, and organizational identification. Organizational Behavior and Human Decision Processes, 115(2), 204-213. https://doi.org/10.1016/j.obhdp.2010.11.002

Obra bajo licencia de Creative Commons Atribución 4.0 Internacional (CC BY 4.0).

(c) 2020 Autores. 